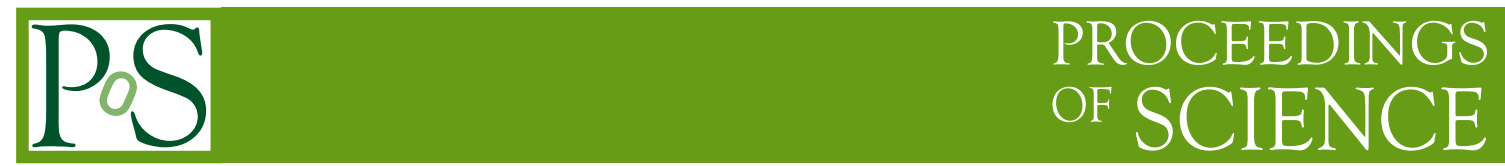

\title{
Virtual Machines \& Volunteer Computing: Experience from LHC@Home: Test4Theory project
}

\author{
Daniel Lombraña González* \\ Citizen Cyberscience Centre \\ E-mail: daniel.lombrana.gonzalez@cern.ch

\section{Francois Grey} \\ Citizen Cyberscience Centre \\ E-mail: Francois.Grey@cern.ch

\section{Jakob Blomer} \\ CERN \\ E-mail: Jakob.Blomer@cern.ch
}

\section{Predrag Buncic}

CERN

E-mail: Predrag.Buncic@cern.ch

Artem Harutyunyan

CERN

E-mail: artem.harutyunyan@cern.ch

\section{Miguel Marquina}

CERN

E-mail: miguel.marquina@cern.ch

\section{Ben Segal}

CERN

E-mail: B.Segal@cern.ch

\section{Peter Skands}

CERN

E-mail: Peter.Skands@cern.ch

\section{Anton Karneyeu}

CERN

E-mail: Anton.Karneyeu@cern.ch 
Volunteer desktop grids are nowadays becoming more and more powerful thanks to improved high end components: multi-core CPUs, larger RAM memories and hard disks, better network connectivity and bandwidth, etc. As a result, desktop grid systems can run more complex experiments or simulations, but some problems remain: the heterogeneity of hardware architectures and software (library dependencies, code length, big repositories, etc.) make it very difficult for researchers and developers to deploy and maintain a software stack for all the available platforms. In this paper, the employment of virtualization is shown to be the key to solve these problems. It provides a homogeneous layer allowing researchers to focus their efforts on running their experiments. Inside virtual custom execution environments, researchers can control and deploy very complex experiments or simulations running on heterogeneous grids of high-end computers. The following work presents the latest results from CERN's LHC@home Test4Theory project, the first BOINC project using virtualization technology, where all the simulations are run inside virtual machines on a wide variety of volunteers' platforms.

The International Symposium on Grids and Clouds (ISGC) 2012,

February 26 - March 2, 2012

Academia Sinica, Taipei, Taiwan

*Speaker. 


\section{Introduction}

LHC@ home started as an outreach project for CERN's 50th Anniversary in 2004. The project calculated the stability of proton orbits using the software Sixtrack [25] in CERN's new LHC accelerator and managed to engage about 60000 active users, over 100000 desktop computers and 3000 CPU years of processing [10] thanks to the BOINC middleware [4].

Due to the success of the project, a challenge was issued: Why don't you run "real" LHC physics on BOINC? However, running real LHC physics simulation code in volunteer commodity computers is extremely complicated due to several factors.

Volunteer computers are one of the most heterogeneous resources for running any type of scientific experiments because they feature different: (i) operating systems : Microsoft Windows, GNU/Linux, or Mac OS X, (ii) hardware architectures : x86, PowerPC, ARM, 32 and 64 bit architectures, nVidia and ATI GPUs, (iii) versions of libraries, applications, operating systems, APIs, etc.

In order to ease the access to such heterogeneous resources, BOINC provides an API and a set of wrappers that help to support the above mentioned differences between hardware and software components in volunteer computers.

While the API and the wrapper approach can improve the situation, BOINC does not solve the problem without further cost. Using the API means that researchers have to either port their existing applications to the supported BOINC programming languages $(\mathrm{C} / \mathrm{C}++$, Fortran and Java), or start from scratch a new version of the application, or provide a statically linked version of the application that could be used with one of the provided wrappers.

While Microsoft Windows is the most popular volunteer platform OS [13], Mac OS X [5] and GNU/Linux systems are growing fast. In any case, the mandatory platform to be supported is Microsoft Windows, including its multiple variants.

Unfortunately, the vast majority of LHC experiments' code only runs on Unix or GNU/Linux systems. Furthermore, the LHC experiments have very large code repositories of libraries, applications, modules, etc. which are updated often. Therefore, porting all the source code to Windows machines in order to run them in BOINC would be quite impractical.

Another important point is that the physicists have built their own facilities and systems for handling their queues of batch jobs, and adopting a new tool like BOINC for this purpose would be very difficult and unpopular.

A solution to these problems is to use virtualization technology [28]. In a guest virtual machine (VM) it is possible to load an OS and all its software applications. The VM is handled by a Virtual Machine Monitor (VMM) or hypervisor which works at the kernel level of the host machine. This technology provides a homogeneous application layer across all the described platforms and OSes. Moreover, it also provides:

- Resource isolation. Virtualization isolates each VM inside the host machine. In other words, a failure in the guest machine, the VM, does not affect the host machine, creating a safe sandbox for running the experiments' applications.

- Guest OS instantiation. The instantiation facility permits creation of VM images that can be deployed on other hosts with the same hypervisor. This allows us to support all desktop 
PC OSes by simply installing the same hypervisor on them.

- Snapshots or state serialization (also known as checkpointing). The guest VM can be paused and restarted at any point of its execution. This feature will allow us to support the typical host churn behaviour (volunteers powering on/off their computers, thus stopping execution of their BOINC applications) by saving the latest execution state of the VM and resuming it at a later stage.

\section{Virtualization at CERN}

In 2008, a CERN R\&D project called CernVM [8] was launched by Predrag Buncic and collaborators in the CERN PH Department, offering a general solution to the problem of virtual machine image management for physics computing at the LHC experiments. CernVM provides the required technologies that allow running real LHC experiment code [15] in desktop computers. Its heart is a minimum virtual machine "appliance" - less than $250 \mathrm{MB}$ in size - that will download by itself all the required libraries for LHC applications, and also provide a secure gateway to the experiments' own job management systems.

CernVM supports different hypervisors: VirtualBox [32], VMware [29], Xen [6] and KVM [17]. Xen and KVM only work in GNU/Linux systems, so they cannot be employed in BOINC due to the need to support all desktop OSes. VMware and VirtualBox do support all the OSes: Windows, GNU/Linux and Mac OS X; however VMware is not open source, making it more difficult to distribute the software among the volunteers in comparison with VirtualBox which is open source.

Therefore CernVM virtual machines, in combination with the VirtualBox open source hypervisor, were chosen as the solution for all the problems involved in running LHC code in volunteer PCs. BOINC provides the volunteer community support, and the facilities to distribute these virtual machines. We need only one extra step to support this new type of BOINC project: the installation of VirtualBox as well as the standard BOINC client.

Using virtualization for running the experiments will add some overhead, however from the point of view of the experiments it is negligible [12]. Virtualization hypervisors have been designed and implemented to reduce as much as possible the overheads caused in the virtualized environment. In general, the overhead is less than a few percent in CPU and network operations, while it has bit more of impact in I/O operations [6, 32, 17, 12].

Nowadays modern microchips include a specific set of instructions (Intel Extended Page Tables [31] or AMD Rapid Virtualization Indexing [30]) just designed for virtualization performance improvements in the $\mathrm{x} 86$ platform with gains between 42 and $48 \%$ MMU-intensive benchmarks and 500 to $600 \%$ in MMU-intensive microbenchmarks.

Therefore, the benefits of running any application in any platform without adapting or porting the source code are valuable, as deploying and running new experiments can be carried out easily with a minimum cost in terms of overhead.

After a joint effort by the CernVM team and several summer students, the first BOINC project using CernVM in virtual machines running under the open source virtualization hypervisor VirtualBox was born: the LHC@home Test4Theory project [11]. 


\subsection{Linking CernVM and BOINC}

CernVM provides the virtual machine that will be downloaded by the BOINC clients; however when the project started there was no solution within the BOINC framework that supported virtual machines. Therefore a new type of BOINC wrapper was created to support this feature: the CernVM Wrapper [19].

The Virtual Machine (VM) is a thin appliance of about $200 \mathrm{MB}$ which is only loaded initially with the minimum required system to boot the VM. This approach reduces the impact of downloading a full VM containing all the code and libraries for an experiment (typically around $10 \mathrm{~GB}$ in size). The thin appliance via the CernVM File System [7] will download and cache the specific binary libraries and code for the experiment from a CERN software repository.

The CernVM-FS provides a scalable hierarchy of standard HTTP caches ${ }^{1}$ (content-addressable storage, content delivery networks, local and distributed memory caching) that allow the delivery of scientific software to globally distributed computing resources including "volunteer clouds" [16]. The file system ensures data integrity and provides full tolerance.

Thanks to the employment of CernVM-FS, the resulting working images are typically under 1 GB in size. CernVM-FS also assures that an updated version of LHC experiment libraries are used within the VMs, using a cache to minimize the access to the repositories.

The CernVM Wrapper is a modified version of the official BOINC wrapper but adapted for VirtualBox deployment. The wrapper is in charge of uncompressing the VM after it has been downloaded to the client, registering the VM in the hypervisor, and launching it. It will also pause or resume the $\mathrm{VM}$ when the BOINC associated task has to be paused or resumed to follow the standard work flow of BOINC. This solution does not modify in principle the standard BOINC project model.

The wrapper is open source, written in C++ and publicly available in a Github repository [19]. While the wrapper mimics the same behavior as any other standard BOINC task, there is a subtle difference: the job distribution is carried out via the CernVM appliance and not by the BOINC framework, which means that BOINC does not send or receive LHC jobs at all. From the point of view of the BOINC core client, its job is to download a VM as an input file for the CernVM Wrapper task, and run it. The wrapper then runs the VM for typically 24 hours, allowing it to perform different LHC simulation jobs during this fixed period. See Figure 1 for more details about this architecture [26].

The CERN LHC jobs are distributed via the Co-Pilot agent [14] which interfaces to the LHC experiment's own Pilot-job systems, offering a gateway to these different Pilot job implementations. Figure 2 shows the Co-Pilot infrastructure. In fact, the work flow of jobs is handled by CernVM and Co-Pilot, on behalf of the physicists at CERN, making no distinction between volunteer computing nodes or other nodes running CernVM [15]. All such CernVM nodes are interfaced as a Cloud, with the BOINC nodes thus forming a "Volunteer Cloud" [26] .

The following section will give more details about the project Test4Theory and the actual results that have been achieved so far.

\footnotetext{
${ }^{1}$ For further details about the CernVM-FS check [16].
} 


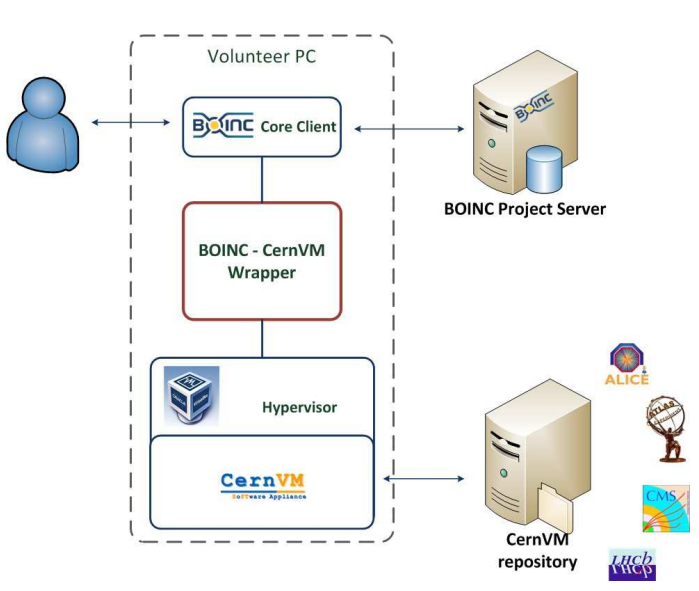

Figure 1: BOINC \& CernVM

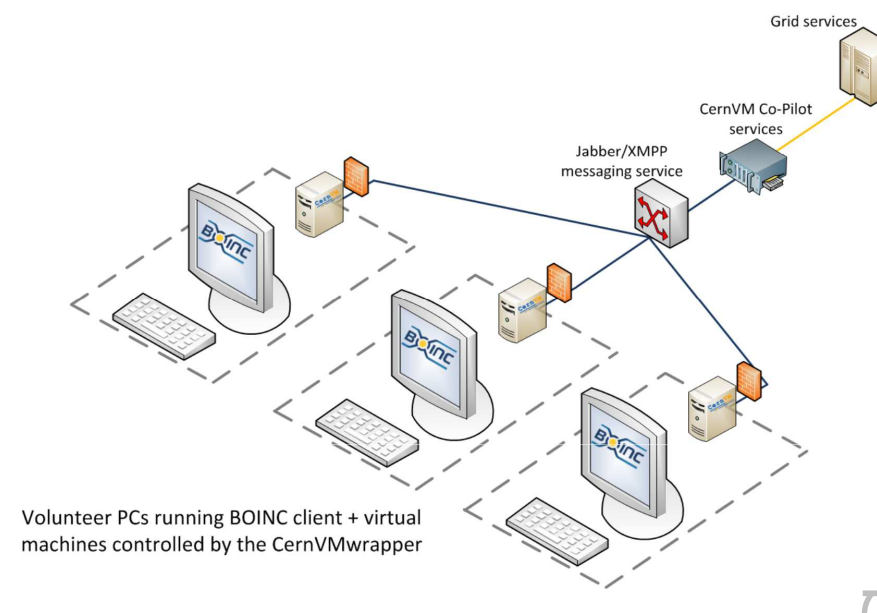

Figure 2: BOINC \& CernVM \& Co-Pilot

\begin{tabular}{|l|c|c|c|c|}
\hline & 7 of March of 2012 & Week 14 of 2012 & March of 2012 & Total \\
\hline Submitted & 21686 & 151802 & 715611 & 3975036 \\
\hline Queue drain & 20683 & 145110 & 706594 & 3961143 \\
\hline Received & 18938 & 133135 & 642351 & 3398421 \\
\hline Succeeded & 14528 & 103250 & 455475 & 2589328 \\
\hline Failed & 4410 & 29885 & 186876 & 809093 \\
\hline \multicolumn{4}{|c|}{ Total number of generated events } & 257.95 billions \\
\hline
\end{tabular}

Table 1: Test4Theory job statistics

\section{Test4Theory}

The project has now attracted almost 12000 volunteers ${ }^{1}$ with more than 15000 PCs $^{1}$ registered (see Figures 3 and 4) who have already generated more than 250 billion events $^{2}$ (see Table 1) for the Monte Carlo QCD event generation project, MCPLOTS [27].

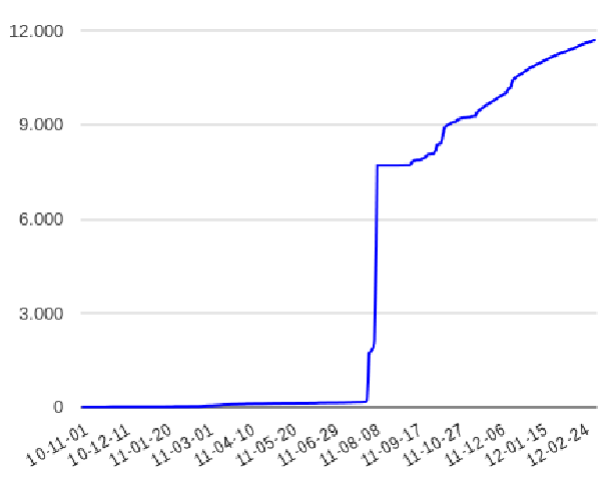

Figure 3: Total number of users per day

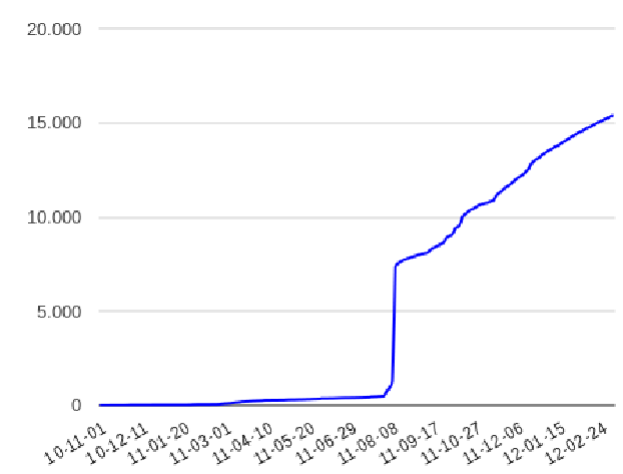

Figure 4: Total number of hosts per day

\footnotetext{
${ }^{1}$ All the data can be checked on-line, see [1].

${ }^{2}$ All the data can be checked on-line, see [27].
} 


\subsection{Community Management}

In a volunteer computing project, the community is all. Volunteers participate in these types of projects because they can feel part of the research effort. In return they want some acknowledgment, but also some feedback from the project and the achievements that have been obtained thanks to the CPU cycles they are donating. BOINC provides several channels for communication, both among volunteers and with the project team.

The Test4Theory team has been interacting strongly with the community, notifying them of new releases, improvements, etc., answering their questions, requesting feedback about how to improve their volunteer experience, and channeling specific questions (such as physics questions about the simulations) to the right persons in the project team.

At the beginning of the project there was only a minimum number of expert users (25), as the project was in the very early stages, and volunteers could only join the project by invitation. The project fully opened its doors to the general public on August 8th, 2011 with an official CERN Press Release [9]. That day more than 2000 new users and 1000 new computers were registered in the project (see on-line charts [1] for real data). Three days later the project reached the amount of more than 7000 thousand users and computers attached to the project.

The CERN press release was a very powerful trigger, and the article spread over the Web to all the big on-line media magazines (Wired, Engadget, Discovery, BBC, MSNBC, Ars-Technica, etc. [2]).

This massive attention and new registrations caused several problems to the infrastructure, and the project had to reactivate the invitation codes to manage all the new requests, and to have time to focus on fixing the problems.

Before opening again, the project activated a form where users could register themselves to get an invitation code and join the project. This measure was taken to increase the number of users in the project in a controlled way. The form was activated at the end of August and it worked until mid November of 2011. During this period more than 3000 users registered their email to get an invitation (see Figure 5), having peaks the first days of over 200 new petitions.

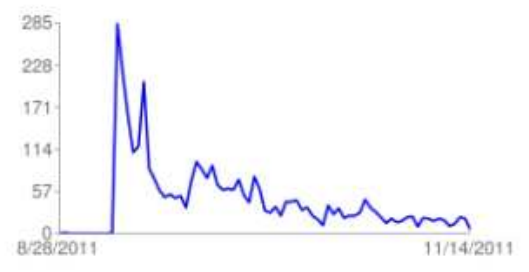

Figure 5: Invitation code registrations per day

By the 14th of November the project was fully stable again, and since then the invitation code has been removed allowing anyone to join the project at any moment.

The main community meeting point is via the message boards. There are four main different forums: News, Science, Number Crunching and Questions \& Answers. "News" is basically used as a feed for publishing news about the project. "Science" is devoted to comments, ideas, and achievements about the research topics of the project. While this message board is usually used 
for communicating news from the project related to the research, the community itself is using this space to ask questions to the researchers about the simulations, and in general any interesting topic that comes from CERN. The activity of this board is usually low, but the topics discussed are really focused on the science behind the project.

Most of the activity is usually gathered in the Number Crunching forum. "Number Crunching" is full of questions, suggestions, feedback, bugs, etc. from the community and it has become one of the most useful documentation sources for the volunteers. The reason is that most of the documentation has been written by the volunteers, creating "sticky" threads at the top of the forum that help new volunteers to answer the frequently asked questions about the project. "Questions \& Answers" is a more focused version of Number Crunching but with different sub-forums for every platform: Windows, GNU/Linux and Mac OS X.

Since the beginning of the project, almost 10000 posts (see Figure 6) have been written in the message boards [1] keeping the community active. Several days were very active (more than 60 messages per day, see Figure 7), usually matching the release of new versions of the system or a public announcement ( 3 days after the CERN press release 215 posts were written in the forums) see Figure 7. All this data can be checked on-line [1].

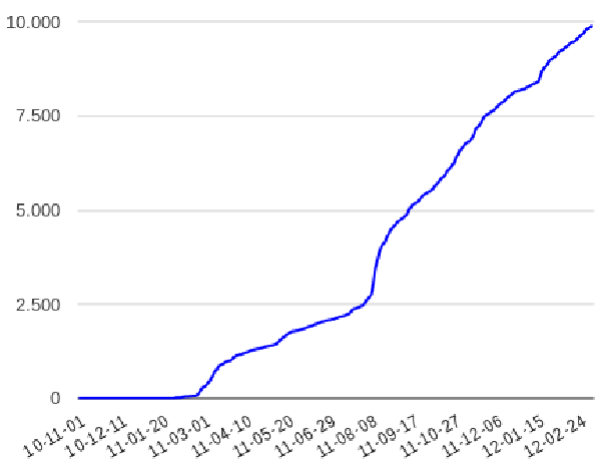

Figure 6: Number of total posts per day

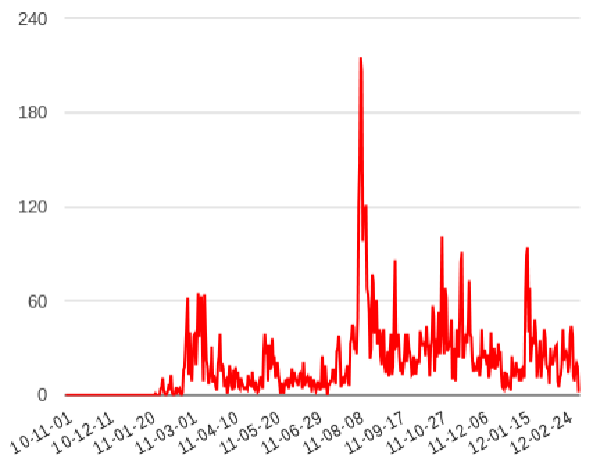

Figure 7: Number of new posts per day

The communication between the community and the researchers and developers has created a good atmosphere in the message boards, and a good response within the community. Here is a sample of some of the comments posted by members of the community in the project's Message Boards about how they like to be involved and updated from the project side:

Crystal Pellet (full message [23]):

"Most other BOINC-projects can follow an example of how the T4T-team is bothering to get the volunteers involved." 
Rechenkuenstler (full message [24]):

"thank you very much for all the effort, that you do with your hard development work. And also let me thank you for all the care that you provide for us volunteers. I'm also crunching in other projects, but the contact to the developers and scientists is the very best here at T4T."

\subsection{New tools for the project}

Based on the feedback of the volunteers, the project has been focused in providing the right tools to have them engaged, and to get more volunteers involved. One of the most important aspects for many volunteers is the visualization of the results that they have produced, as well as the efficient utilization of the resources they contribute to the project.

Since the beginning of the project, some volunteers were asking for a multi-core solution, as modern PCs feature two or more cores. The multi-core feature has been released in the beginning of January of 2012.

Three different tools have been created to show data visualizations for the project:

- The BOINC widgets is a web application integrated within the BOINC web pages, that draws real time line charts (using the Google Chart API) showing the numbers of users, hosts or posts in the message forums.

- The Volunteers map is a web application that shows in a Google Map where are the volunteers all over the world contributing CPU cycles for the project.

- The Web Application is distributed within the virtual machine and provides a user friendly interface, via the volunteer's web browser, to data displays and logs produced by the simulation job currently running in the virtual machine.

The next sections give more details about each of these applications.

\subsubsection{The multi-core Virtual Machine}

At the beginning of the project, Jie $\mathrm{Wu}$, a CERN summer student, developed the first version of the CernVM wrapper. This initial version registered the virtual machine and ran it for the project using as base code the general purpose wrapper code from the BOINC community. While the new CernVM wrapper worked, there was a problem: BOINC was not designed to run Virtual Machines. Thus, further wrapper development has had to incorporate new functionalities or "hacks" that solve some of these issues: for instance the multi-core support for VMs. Such newly discovered requirements to run VMs within BOINC were communicated to the main developers of BOINC to include them in future releases of the client.

Multi-core support has become one of the main features of the project, as the Test4Theory simulations can use up to two cores. Looking ahead to other LHC simulations, this solution supports up to 64 cores in BOINC and VirtualBox out of the box (respecting user preferences), making it possible to run very complex simulations and multi-threaded or MPI applications within the VM without problems. Additionally, the volunteers can specify CPU throttling to be used in the VM as well as the number of cores they want to donate via the BOINC user preferences interface. 
All the described items can be publicly reviewed in the official repository. Please check the source code, the change log, and documentation about this development [19].

\subsubsection{Web Application}

The Test4Theory community asked our developers several times to see some output of the simulations that their computers were processing. While there is a BOINC API to support an application graphics window showing users output from a BOINC task running in the host, the use of VMs in this BOINC project makes it very difficult, as by default every VM is a secure sand-box. Hence, accessing the data within the VM is not straightforward. Nevertheless, a standard BOINC graphics application was developed by a summer student in 2011 [22].

Our preferred solution uses the benefits of having a custom execution environment where it is possible to install any application or software. Thanks to this freedom, a web server is installed in the VM with a web application that shows the users information about the jobs running inside their $\mathrm{VMs}^{2}$ as well as some plots generated by these simulations.

The benefits of using this approach are:

- it will run in all platforms as it uses a web page, not a special application window,

- it will be possible to deploy new versions without requiring user intervention (thanks to the CernVM file system [7]),

- in future it will be possible to develop web browser extensions, improving in general the user experience.

The web application ${ }^{3}$ (see Figure 8 ) has been created using the latest open source technologies for web development: HTML5, CSS3 and JavaScript. The employment of these technologies have enabled us to create a user friendly interface to the experiments, with a video tutorial that interacts with the user interface (hiding and showing elements, adding pop-ups, etc. in sync with the video), using only open web standards (i.e. the video uses the open source code WebM [3] which is a royalty-free and open video codec for the web supported by companies like Google, Mozilla, Adobe, etc.).

The video tutorial offers an attractive and flexible tool to explain the user interface to the volunteers, and new videos will be created to explain the results obtained by the simulations, so volunteers can have a better understanding of the science behind the project.

\subsubsection{Volunteers Map}

In order to view the distribution of the volunteers all over the world, the project uses the logs from the Co-Pilot agent to geo-locate the VMs via their IP address and plot them in a Google Map over the last 24 hours. See Figure 9 or try it on-line [11]. The map right now only shows the Co-Pilot ID given to the user, but a new method is being implemented to show the BOINC user name, so volunteers can find themselves in the map.

\footnotetext{
${ }^{2}$ The web application is exposed by NAT forwarding to the host.

${ }^{3}$ The source code is open source and published in Github [20]. Check the demo site for more details [21].
} 


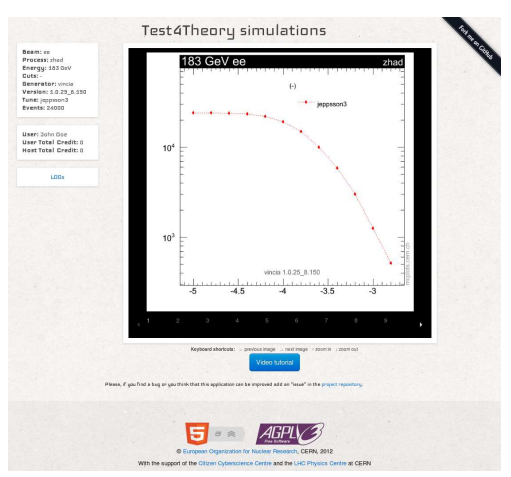

Figure 8: T4T web application

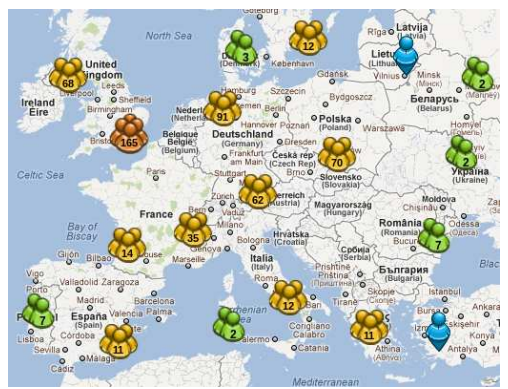

Figure 9: Volunteer's map

\subsubsection{BOINC Widgets}

BOINC provides access to its database in a raw format. Understanding and looking for trends is complicated, as usually one has to run SQL queries to get the requested data, and later process it using a different tool. For this reason, the project has developed a set of visualization tools widgets - that allow us to display the number of users, hosts or message board posts over a period of time. These widgets can be integrated with any other BOINC project as they use the standard server API from BOINC.

The widgets have been created using the Google Charts API, a third-party solution that allows one to develop fast and rich statistics dashboards [18]. For instance, see Figures 10, 11 for user statistics ${ }^{4}$.

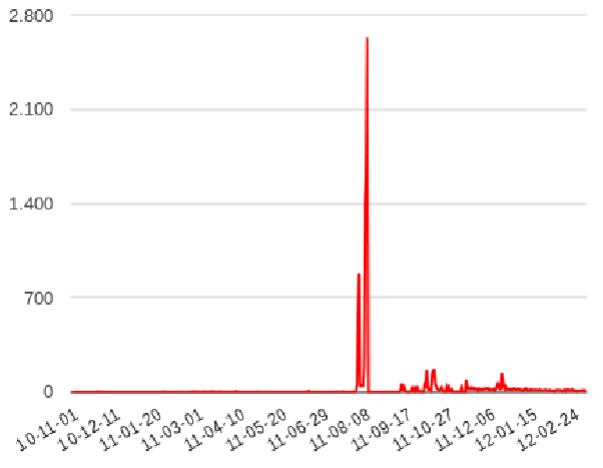

Figure 10: New registered users per day

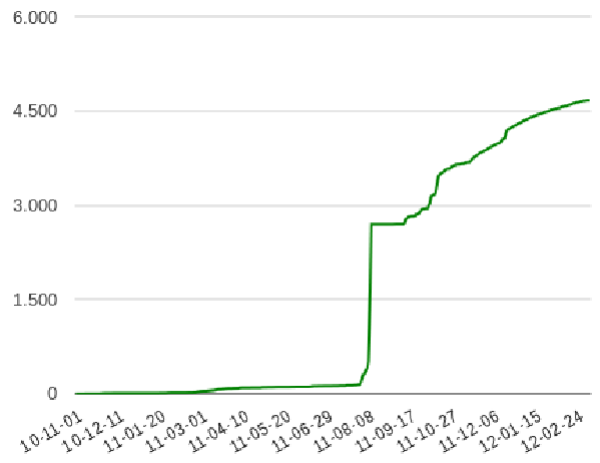

Figure 11: Users with credit per day

\section{Conclusions}

Test4Theory is the first project in the BOINC community using virtual machines to run complex physics simulations in home desktop computers all over the world. The system has been tested for more than a year, generating more than 250 billion events thanks to volunteers. Due to this success the official BOINC development team is integrating this new technology in their framework, thanks to a collaboration of both the projects.

\footnotetext{
${ }^{4}$ All the data can be checked on-line, see [1].
} 
The benefits of using a virtualization layer - sand-boxing, custom execution environments, checkpointing out of the box - improve the user experience for both developers and volunteers. Developers can develop for only one platform, reducing the costs of porting the code to all available PC architectures and OSes, and reaping the benefits of creating custom execution environments tailored for their specific needs. For the volunteers, they know that if there is a bug, their systems will be protected from any damage as the sand-boxing provided by virtualization will only affect the guest VMs and not their hosts.

\section{Future work}

In the coming months, our project team will be working to adopt the latest BOINC source code for the server, client and virtualization wrapper modules, developed and supported by the official BOINC team. This migration should not affect the user experience, as the required virtualization features have been included in this new version of BOINC thanks to a collaboration between both projects.

Our project team will also continue working on extending the web applications, adding new features, tutorials, and an add-on for the open source web browser Firefox, that will give the users the chance to have an integrated experience with the project directly from their browsers.

\section{Acknowledgments}

To our Test4Theory community for all their feedback, patience, help, complaints, ..., since the beginning of the project.

\section{References}

[1] Test4Theory BOINC widgets with statists about the project, Online 2012. http://Ihcathome2.cern.ch/test4theory/project_stats_charts.php.

[2] Test4Theory project in the internet media, Online 2012. http: // Ihcathome2.cern.ch/media.

[3] The webm project. Technical report, Google, Online 2012. http: / / www . webmpro ject . org/.

[4] D. Anderson. BOINC: a system for public-resource computing and storage. In 5th IEEE/ACM International Workshop on Grid Computing, pages 4-10, 2004.

[5] Apple. Apple reports third quarter results, Online 2012. http: / /www . apple.com/pr/ library/2011/07/19Apple-Reports-Third-Quarter-Results.html.

[6] P. Barham, B. Dragovic, K. Fraser, S. Hand, T. Harris, A. Ho, R. Neugebauer, I. Pratt, and A. Warfield. Xen and the art of virtualization. Proceedings of the nineteenth ACM symposium on Operating systems principles, pages 164-177, 2003.

[7] J. Blomer and T. Fuhrmann. A fully decentralized file system cache for the CernVM-FS. Computer Communications and Networks (ICCCN), (19):1-6, 2010.

[8] P. Buncic, C. A. Sanchez, J. Blomer, L. Franco, A. Harutyunian, P. Mato, and Y. Yao. CernVM - a virtual software appliance for LHC applications. Journal of Physics: Conference Series, 219(4):042003, 2010. 
[9] CERN. CERN supports european year of volunteering through citizen cyberscience centre. CERN Press Release, Online 2011. http:

//press.web. cern. ch/press/pressreleases/Releases2011/PR13.11E.html.

[10] CERN. LHC@Home: Sixtrack web project, Online 2012.

http://lhcathomeclassic.cern.ch/sixtrack/.

[11] CERN. LHC@Home: Test4Theory, Online 2012.

http://1hcathome2. cern. ch/test4theory/.

[12] U. Drepper. The cost of virtualization. Queue, 6:28-35, January 2008.

[13] Gartner. Gartner says windows 7 will be running on 42 percent of PCs in use worldwide by the end of 2011, Online 2012. http: / / www . gartner. com/it/page. jsp?id=1762614.

[14] A. Harutyunyan, C. Aguado-Sanchez, J. Blomer, and P. Buncic. CernVM CoPilot: a framework for orchestrating virtual machines running applications of lhc experiments on the cloud. Journal of Physics: Conference Series, 331, 2011.

[15] A. Harutyunyan, P. Buncic, T. Freeman, and K. Keahey. Dynamic virtual AliEn grid sites on Nimbus with CernVM. Journal of Physics: Conference Series, 219(7):072036, 2010.

[16] Jakob Blomer and Predrag Buncic and Thomas Fuhrmann. CernVM-FS: delivering scientific software to globally Distributed computing resources. pages 49-56, 2011.

[17] A. Kivity, Y. Kamay, D. Laor, U. Lublin, and A. Liguori. KVM: the linux virtual machine monitor. In Proc. of the 2007 Linux Symposium, pages 225-230, 2007.

[18] D. Lombraña-González. BOINC widgets. Technical report, Citizen Cyberscience Centre, Online 2012. https://github.com/teleyinex/boinc-widgets.

[19] D. Lombraña-González. CernVM wrapper. Technical report, Citizen Cyberscience Centre, Online 2012. https://github.com/citizen-cyberscience-centre/cernvmwrapper.

[20] D. Lombraña-González. t4t-webapp. Technical report, Citizen Cyberscience Centre, Online 2012. https://github.com/citizen-cyberscience-centre/t4t-webapp.

[21] D. Lombraña-González. t4t-webapp. Technical report, Citizen Cyberscience Centre, Online 2012. http://www.citizencyberscience.net/t4t-webapp/.

[22] B. Page. Test4Theory CernVM graphics application, Online 2012. https://github.com/Volatilestorm/CernVM-Graphics.

[23] C. Pellet. Community feedback in the message boards of Test4Theory, Online 2012. http://goo.gl/bffMf.

[24] Rechenkuenstler. Community feedback in the message boards of Test4Theory, Online 2012. http://goo.gl/BGFPI.

[25] F. Schmidt. SIXTRACK: a single particle tracking code. oai:cds.cern.ch:212031. (CERN-SL-90-98-AP):12 p, Sep 1990.

[26] B. Segal and et al. LHC Cloud computing with CernVM. Proceedings of the XIII International Workshop on Advanced Computing and Analysis Techniques in Physics Research (ACAT10), Jaipur, 2010.

[27] P. Skands, A. Karneyeu, D. Konstantinov, M. Mangano, L. Mijovic, W. Pokorski, S. Prestel, and A. Pytel. MC production statistics. Technical report, CERN, Online 2012. http: //mcplots-dev. cern. ch/production.php?view=status. 
[28] J. E. Smith and R. Nair. Virtual Machines. Morgan Kaufmann, 2005.

[29] J. Sugerman, G. Venkitachalam, and B. Lim. Virtualizing I/O devices on VMware workstation's hosted virtual machine monitor.

citeseer.ist.psu.edu/venkitachalam01virtualizing.html.

[30] VMware. Performance evaluation of amd rvi hardware assist.

[31] VMware. Performance evaluation of intel ept hardware assist.

[32] J. Watson. VirtualBox: bits and bytes masquerading as machines. Linux J., 2008(166):1, 2008. 